\title{
SUMMARIZING SETS OF CATEGORICAL SEQUENCES Selecting and visualizing representative sequences
}

\author{
Alexis Gabadinho, Gilbert Ritschard, Matthias Studer, Nicolas S. Müller \\ Department of Econometrics and Laboratory of Demography, University of Geneva \\ 40, bd du Pont-d'Arve, CH-1211 Geneva, Switzerland \\ \{alexis.gabadinho, gilbert.ritschard, matthias.studer, nicolas.muller\}@unige.ch
}

Keywords: Categorical sequence data, Representativeness, Dissimilarity, Discrepancy of sequences, Summarizing sets of sequences, Visualization.

\begin{abstract}
This paper is concerned with the summarization of a set of categorical sequence data. More specifically, the problem studied is the determination of the smallest possible number of representative sequences that ensure a given coverage of the whole set, i.e. that have together a given percentage of sequences in their neighborhood. The goal is to yield a representative set that exhibits the key features of the whole sequence data set and permits easy sounded interpretation. We propose an heuristic for determining the representative set that first builds a list of candidates using a representativeness score and then eliminates redundancy. We propose also a visualization tool for rendering the results and quality measures for evaluating them. The proposed tools have been implemented in TraMineR our $\mathrm{R}$ package for mining and visualizing sequence data and we demonstrate their efficiency on a real world example from social sciences. The methods are nonetheless by no way limited to social science data and should prove useful in many other domains.
\end{abstract}

\section{INTRODUCTION}

Sequences of categorical data appear in many different scientific fields. In the social sciences, such sequences are mainly ordered list of states (employed/unemployed) or events (leaving parental home, marriage, having a child) describing individual life trajectories, typically longitudinal biographical data such as employment histories or family life courses.

One widely used approach for extracting knowledge from such sets consists in computing pairwise distances by means of sequence alignment algorithms, and next clustering the sequences by using these distances. This method has been applied to various data since the pioneering work of (Abbott and Forrest, 1986). A review can be found in (Abbott and Tsay, 2000). The expected outcome of such a strategy is a typology, with each cluster grouping cases with similar patterns (trajectories).

An important aspect of sequence analysis is also to compare the patterns of cases grouped according to the values of covariates (for instance sex or socioeconomic position in the social sciences).

A crucial task is then to summarize groups of sequences by describing the patterns that characterize them. This could be done by resorting to graphical representations such as sequence index plots, state distribution plots or sequence frequency plots (Müller et al., 2008). However, relying on these graphical tools suffers from some drawbacks. Sequence index plots give a (sorted) view of all the sequences in each subset and assigning them a meaning is mainly a subjective task. State distribution plots are aggregated transversal views that occult individual sequences and their interpretation can be misleading. Sequence frequency plots that focus on the most frequent sequences provide only a partial view especially when there is a great number of distinct sequences.

Hence, we need more appropriate tools for ex- 
tracting the key features of a given subset or data partition. We propose an approach derived from the concept of representative set used in the biological sciences (Hobohm et al., 1992; Holm and Sander, 1998). The aim in this field is mainly to get a reduced reference base of protein or DNA sequences for optimizing the retrieval of a recorded sequence that resembles to a provided one. In this setting, the representative set must have "maximum coverage with minimum redundancy" i.e. it must cover all the spectrum of distinct sequences present in the data, including "outliers".

Our goal is similar regarding the elimination of redundancy. It differs, however, in that we consider in this paper representative sets with a user controlled coverage level, i.e. we do not require maximal coverage. We thus define a representative set as a set of non redundant "typical" sequences that largely, though not necessarily exhaustively covers the spectrum of observed sequences. In other words, we are interested in finding a few sequences that together summarize the main traits of a whole set.

We could imagine synthetic - not observed typical sequences, in the same way as the mean of a series of numbers that is generally not an observable individual value. However, the sequences we deal with in the social sciences (as well as in other fields) are complex patterns and modeling them is difficult since the successive states in a sequence are most often not independent of each other. Defining some virtual non observable sequence is therefore hardly workable, and we shall here consider only representative sets constituted of existing sequences taken from the data set itself.

Since this summarizing step represents an important data reduction, we also need indicators for assessing the quality of the selected representative sequences. An important aspect is also to visualize these in an efficient way.

Such tools and their application to social science data are presented in this paper. These tools are new features of our TraMineR library for mining and visualizing sequences in $\mathrm{R}$ (Gabadinho et al., 2009).

\section{DATA}

To illustrate our purpose we consider a data set from (McVicar and Anyadike-Danes, 2002) stemming from a survey on transition from school to work in Northern Ireland. The data contains 70
Table 1: List of states in the mvad data set.

\begin{tabular}{|c|c|l|}
\hline 1 & EM & Employment \\
2 & FE & Further education \\
3 & HE & Higher education \\
4 & JL & Joblessness \\
5 & SC & School \\
6 & TR & Training \\
\hline
\end{tabular}

monthly activity state variables from July 1993 to June 1999 for 712 individuals. The alphabet is made of 6 states detailed in Table 1 .

The three first sequences of this data set represented as distinct states and their associated durations (the so called State Permanence Format) look as follows

\section{Sequence}

[1] $\mathrm{EM} / 4-\mathrm{TR} / 2-\mathrm{EM} / 64$

[2] $\mathrm{FE} / 36-\mathrm{HE} / 34$

[3] TR/24-FE/34-EM/10-JL/2

We consider in this paper the outcome of a cluster analysis of the sequences based on Optimal Matching (OM). The OM distance between two sequences $x$ and $y$, also known as edit or Levenshtein distance, is the minimal cost in terms of indels - insertions and deletions - and substitutions necessary to transform $x$ into $y$. We computed the distances using a substitution cost matrix based on transition rates observed in the data set and an indel cost of 1 . The clustering is done with an agglomerative hierarchical method using the Ward criterion. A four cluster solution is chosen. Table 2 indicates some descriptive statistics for each of the clusters.

The sequence frequency plots in Fig. 1 display the 10 most frequent sequences in each cluster and give a first idea of their content. The bar widths are proportional to the sequence frequencies. The 10 most frequent sequences represent about $40 \%$ of all the sequences in cluster 1 and 2, while this proportion is $27 \%$ and $21 \%$ for clusters 3 and 4 due to a higher diversity of the patterns.

Table 2: Number of cases, distinct sequences and discrepancy within each cluster.

\begin{tabular}{|lrrr|}
\hline & $\mathrm{N}$ & Dist. seq. & Discr. \\
\hline Cluster 1 & 265 & 165 & 18.3 \\
Cluster 2 & 153 & 88 & 23.5 \\
Cluster 3 & 194 & 148 & 27.9 \\
Cluster 4 & 100 & 89 & 37.2 \\
\hline
\end{tabular}



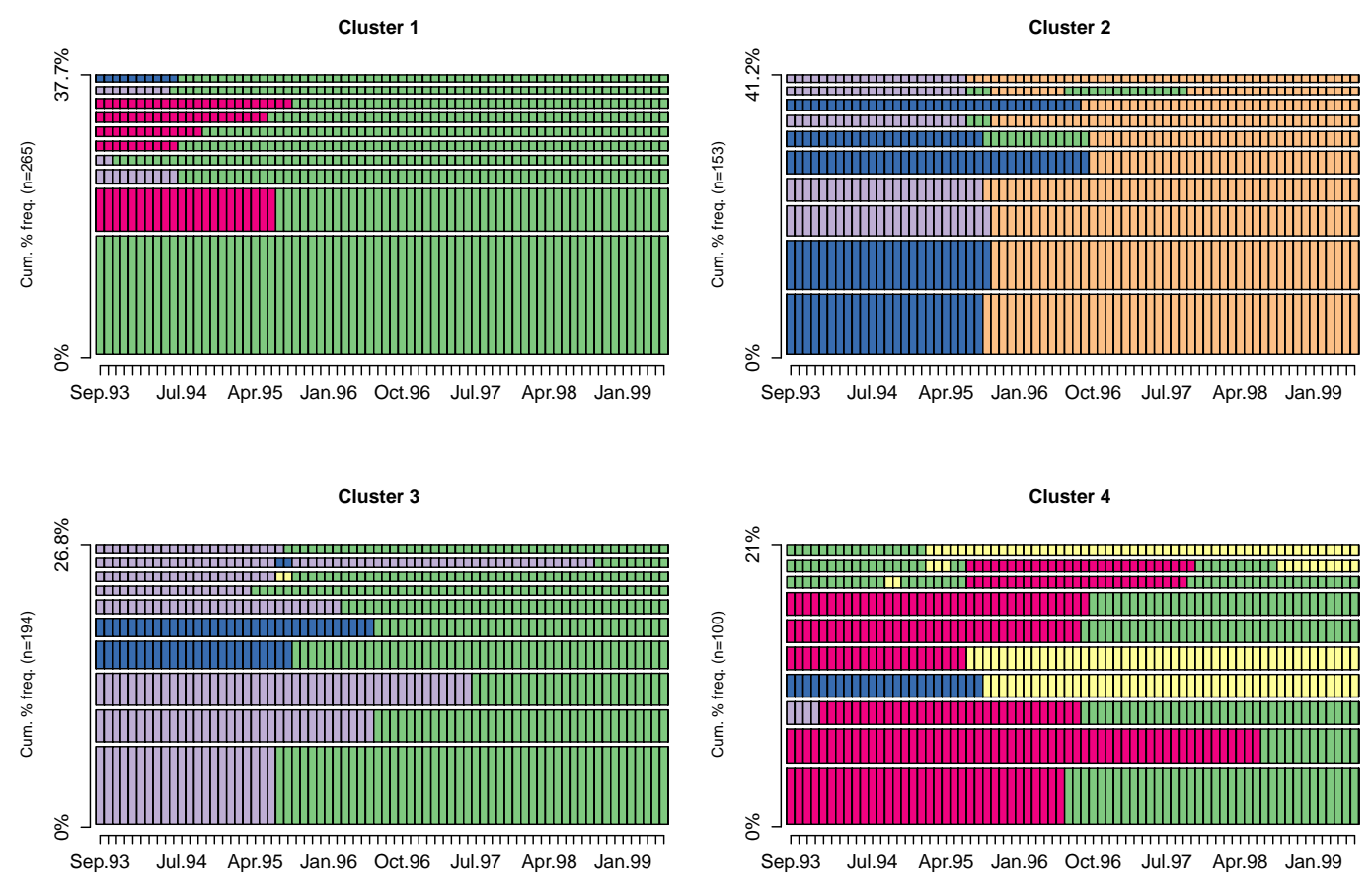

$$
\begin{array}{ll}
\square \text { employment } & \square \text { higher education } \\
\square \text { further education } & \square \text { joblessness }
\end{array} \quad \square \text { training }
$$

Figure 1: 10 most frequent sequences within each cluster.

\section{METHODS}

Our aim is to find a small subset of non redundant sequences that ensures a given coverage level, this level being for instance defined as the percentage of cases that are within a given neighborhood of at least one of the representative sequences. We propose an heuristic for determining such a representative subset.

It works in two main steps. In the first stage it prepares a sorted list of candidate representative sequences without caring for redundancy and eliminates redundancy within this list in a second stage. It basically requires the user to specify a representativeness criterion for the first stage and a similarity measure for evaluating redundancy in the second one.

\subsection{Sorting Candidates}

The initial candidate list is made of all distinct sequences appearing in the data. Since the second stage will extract non redundant representative sequences sequentially starting with the first element in the list, sorting the candidates according to a chosen representativeness criterion ensures that the "best" sequences given the criterion will be included. We present here five alternatives for measuring the sequence representativeness.

Sequence Frequency. A first simple criterion is to sort the sequences according to their frequency. The more frequent a sequence the more representative it is supposed to be. Hence, sequences are sorted in decreasing frequency order.

Neighborhood Density. A second criterion is the number - the density — of sequences in the neighborhood of each candidate sequence. This requires indeed to set the neighborhood diameter. We suggest to set it as a given proportion of the maximal theoretical distance between two sequences. Sequences are sorted in decreasing density order.

Mean state frequency. A third criterion is the mean value of the transversal frequencies of the 
successive states. Let $s=s_{1} s_{2} \cdots s_{\ell}$ be a sequence of length $\ell$ and $\left(f_{s_{1}}, f_{s_{2}}, \ldots, f_{s_{\ell}}\right)$ the frequencies of the states at time $\left(t_{1}, t_{2}, \ldots t_{\ell}\right)$. The mean state frequency is the sum of the state frequencies divided by the sequence length

$$
\operatorname{MSF}(s)=\frac{1}{\ell} \sum_{i=1}^{\ell} f_{s_{i}}
$$

The lower and upper boundaries of $M S F$ are 0 and $1 . M S F$ is equal to 1 when all the sequences in the set are the same, i.e. when there is a single distinct sequence. The most representative sequence is the one with the highest score.

Centrality. A classical representative of a data set used in cluster analysis is the medoid. It is defined as the most central object, i.e. the one with minimal sum of distances to all other objects in the set (Kaufman and Rousseeuw, 1990). This suggests to use the sum of distances to all other sequences, i.e. the centrality as a representativeness criterion. The smallest the sum, the most representative the sequence. It may be mentioned that the most central observed sequence is also the nearest from the 'virtual' true center of the set (Studer et al., 2009).

Sequence likelihood. The sequence likelihood $P(s)$ is defined as the product of the probability with which each of its observed successive state is supposed to occur at its position. Let $s=s_{1} s_{2} \cdots s_{\ell}$ be a sequence of length $\ell$. Then

$$
P(s)=P\left(s_{1}, 1\right) \cdot P\left(s_{2}, 2\right) \cdots P\left(s_{\ell}, \ell\right)
$$

with $P\left(s_{t}, t\right)$ the probability to observe state $s_{t}$ at position $t$. The question is how to determinate the state probabilities $P\left(s_{t}, t\right)$. One commonly used method for computing them is to postulate a Markov model, which can be of various order. Below, we just consider probabilities derived from the first order Markov model, that is each $P\left(s_{t}, t\right)$, $t>1$ is set to the transition rate $p\left(s_{t} \mid s_{t-1}\right)$ estimated across sequences from the observations at positions $t$ and $t-1$. For $t=1$, we set $P\left(s_{1}, 1\right)$ to the observed frequency of the state $s_{1}$ at position 1 . The likelihood $P(s)$ being generally very small, we use $-\log P(s)$ as sorting criterion. The latter quantity is minimal when $P(s)$ is equal to 1 , which leads to sort the sequences in ascending order of their score.

\subsection{Eliminating Redundancy}

Once a sorted list of candidates has been defined, the second stage consists in eliminating redundancy since we do not want our representative set to contain similar sequences. The procedure is as follows:

- Select the first sequence in the candidate list (the best one given the chosen criterion);

- Process each next sequence in the sorted list of candidates. If this sequence is similar to none of those already in the representative set, that is distant from more than a predefined threshold from all of them, add it to the representative set.

The threshold for sequence similarity is defined as a proportion of the maximal theoretical distance. For the OM distance this theoretical maximum is for two sequences $\left(s_{1}, s_{2}\right)$ of length $\left(\ell_{1}, \ell_{2}\right)$

$D_{\text {max }}=\min \left(\ell_{1}, \ell_{2}\right) \cdot \min \left(2 C_{I}, \max (S)\right)+\left|\ell_{1}-\ell_{2}\right| \cdot C_{I}$

where $C_{I}$ is the indel cost and $\max S$ the maximal substitution cost.

\subsection{Size of the Representative Set}

Limiting our representative set to the mere sequence(s) with the best representative score may lead to leave a great number of sequences badly represented. Alternatively, proceeding the complete list of candidates to achieve a full coverage of the data set is not a suitable solution since we look for a small set of representative sequences.

To control the size of the representative set, we limit the size of the candidate list so that the cumulated frequency of the retained distinct candidates reaches a threshold proportion trep of the whole data set. Setting for instance trep $=25 \%$ ensures that at least $25 \%$ of the sequences will have a representative in their neighborhood and that the final representative set will contain at most $25 \%$ of the distinct sequences of the whole set. Thus trep defines also a minimum coverage level.

There are indeed other possible ways of controlling the size of the representative set such as fixing a) the number or the proportion of sequences in the final representative set, or b) the desired coverage level.

\subsection{Measuring Quality}

A first step to define quality measures for the representative set is to assign each sequence to its nearest representative according to the considered pairwise distances. Let $r_{1} \ldots r_{n r}$ be the $n r$ sequences in the representative set and $d\left(s, r_{i}\right)$ the distance 
between the sequence $s$ and the $i$ th representative. Each sequence $s$ is assigned to its closer representative. When a sequence is equally distant from two or more representatives, the one with the highest representativeness score is selected. Hence, letting $n$ be the total number of sequences and $n a_{i}$ the number of sequences assigned to the $i$ th representative, we have $n=\sum_{i=1}^{n r} n a_{i}$. Once each sequence in the set is assigned to a representative, we can derive the following quantities from the pairwise distance matrix.

Mean distance. Let $S D_{i}=\sum_{j=1}^{n a_{i}} d\left(s_{j}, r_{i}\right)$ be the sum of distances between the $i$ th representative and its $n a_{i}$ assigned sequences. A quality measure is then

$$
M D_{i}=\frac{S D_{i}}{n a_{i}}
$$

the mean distance to the $i$ th representative.

Coverage. Another quality indicator is the number of sequences assigned to the $i$ th representative that are in its neighborhood, that is within a distance $d n_{\max }$

$$
n b_{i}=\sum_{j=1}^{n a_{i}}\left(d\left(s_{j}, r_{i}\right)<d n_{\max }\right) .
$$

The threshold $d n_{\max }$ is defined as a proportion of $D_{\max }$. The total coverage of the representative set is the sum $n b=\sum_{i}^{n r} n b_{i}$ expressed as a proportion of the number $n$ of sequences, that is $n b / n$.

Distance gain. A third quality measure is obtained by comparing the sum $S D_{i}$ of distances to the $i$ th representative to the sum $D C_{i}=$ $\sum_{j=1}^{n a_{i}} d\left(s_{j}, c\right)$ of the distances of each of the $n a_{i}$ sequences to the center of the complete set. The idea is to measure the gain of representing those sequences by their representative rather than by the center of the set. We define thus the quality measure $Q_{i}$ of the representative sequence $r_{i}$ as

$$
Q_{i}=\frac{D C_{i}-S D_{i}}{D C_{i}}
$$

which gives the relative gain in the sum of distances. Note that $Q_{i}$ may be negative in some circumstances, meaning that the sum of the $n a_{i}$ distances to the representative $r_{i}$ is higher than the sum of distances to the true center of the set. A similar measure can be used to assess the overall quality of the representative set, namely

$$
Q=\frac{\sum_{i}^{n r} D C_{i}-\sum_{i}^{n r} S D_{i}}{\sum_{i}^{n r} D C_{i}}=\sum_{i=1}^{n r} \frac{D C_{i}}{\sum_{j} D C_{j}} Q_{i}
$$

Discrepancy. A last quality measure is the sum $S C_{i}=\sum_{j=1}^{n a_{i}} d\left(s_{j}, c_{i}\right)$ of distances to the true center $c_{i}$ of the $n a_{i}$ sequences assigned to $r_{i}$, or the mean of those distances $V_{i}=S C_{i} / n a_{i}$, which can be interpreted as the discrepancy of the set (Studer et al., 2009).

\section{RESULTS}

A graphical tool for visualizing the selected representative sequences together with information measures is included in the TraMineR package. A single function produces a "representative sequence plot" (Figure 2) where the representative sequences are plotted as horizontal bars with width proportional to the number of sequences assigned to them. Sequences are plotted bottomup according to their representativeness score. Above the plot, two parallel series of symbols associated to each representative are displayed horizontally on a scale ranging from 0 to the maximal theoretical distance $D_{\max }$. The location of the symbol associated to the representative $r_{i}$ indicates on axis $A$ the (pseudo) variance $\left(V_{i}\right)$ within the subset of sequences assigned to $r_{i}$ and on the axis $B$ the mean distance $M D_{i}$ to the representative.

\subsection{Key Patterns}

The set of representative sequences found with the sequence frequency criterion is displayed in Figure 2 for each of the four clusters of our example. The plots give clearly a more readily interpretable view of the content of the clusters than the frequency plots displayed in Figure 1. Detailed statistics about these sets are presented in Table 3.

The representative sequences were extracted from a list of candidates sorted in decreasing order of their frequency. The number of candidates was limited by setting the trep threshold for the cumulated frequency of the candidates to $25 \%$. The pairwise distances used are the optimal matching distances that we used for the clustering. The threshold $d n_{\max }$ for similarity (redundancy) between sequences was set as $10 \%$ of the maximal theoretical distance $D_{\max }$. The sequence length being $\ell=70$, the indel cost 1 and the maximal substitution cost 1.9995 , we get $D_{\text {max }}=70 \cdot \min (2,1.9995)=139.96$.

The first cluster is represented by three sequences. The first one, employment during the whole period represents $116(44 \%)$ sequences of the cluster (Table 3), and its neighborhood 
Cluster 1

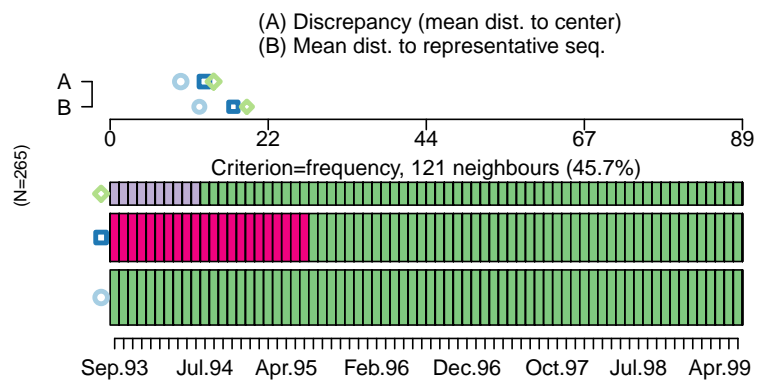

Cluster 3

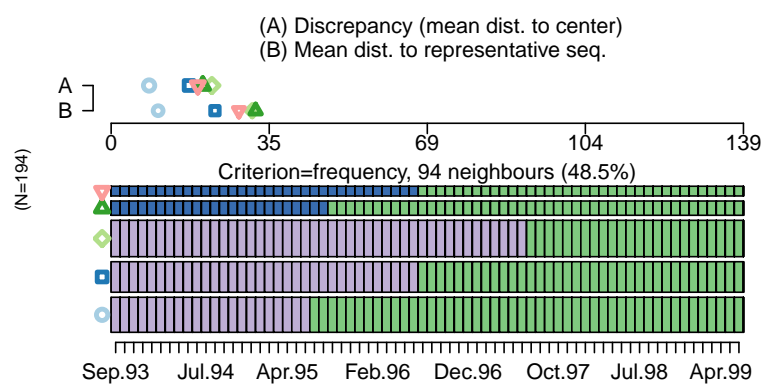

Cluster 2

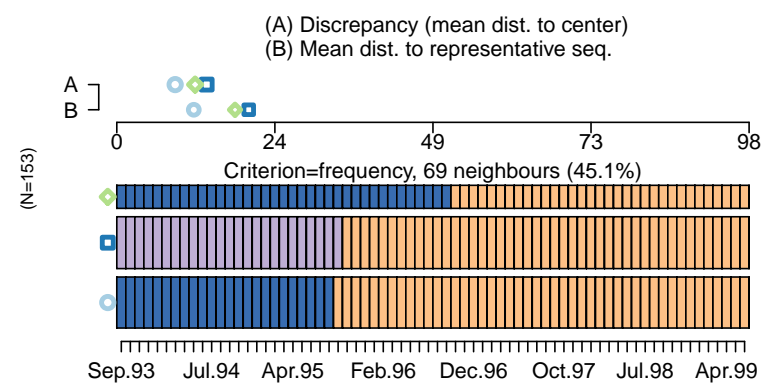

Cluster 4

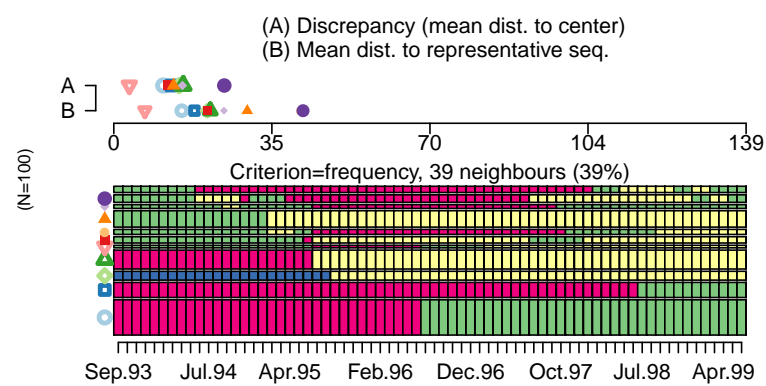

$\square$ employment $\square$ higher education $\square$ school

further education $\square$ joblessness $\square$ training

Figure 2: Representative sequences selected with the Frequency criterion, within each cluster (mvad data).

(within $10 \%$ of $D_{\max }$ ) includes $66(25 \%)$ sequences. The second representative sequence, a spell of training followed by employment represents 101 $(38 \%)$ additional cases and counts $34(13 \%)$ sequences in its neighborhood. The third and last representative sequence exhibits a short spell of further education followed by employment. Hence, this cluster is characterized by patterns of rapid entry into employment. Overall, the distance to the representative is within $10 \%$ of $D_{\max }$ for $121(46 \%)$ sequences of the cluster. The quality measure $Q$ (see Section 3.4) is respectively $11.5 \%, 17 \%$ and $14.5 \%$ for the three sequences in the set and reaches $15 \%$ for the whole set.

The second cluster is described by three patterns leading to higher education, either starting with a spell of further education or with school. These three patterns cover together (have in their neighborhood) $45 \%$ of the sequences and the over- all quality measure for the representative set is $29 \%$.

The number of selected representative sequences in cluster 3 and 4 is higher due to a lesser redundancy in the candidate list. In cluster 3 , the pattern is a transition to employment preceded by long (compared to Cluster 1) spells of school and/or further education. In this cluster, the five representative sequences cover together $48.5 \%$ of the sequences, which is the highest attained value.

The key patterns in cluster 4 was less clear when looking at the sequence frequency plot (Figure 1). This group is dominated by long spells of training leading to employment or joblessness and by disrupted patterns containing spells of joblessness. Hence these trajectories can be characterized as less successful transitions from school to work. The diversity of the patterns is high in this cluster which leads to the extraction of 
Table 3: Representative sequences by cluster, frequency criterion, trep $=25 \%$.

\begin{tabular}{|l|rr|rr|rrr|}
\hline & $n a$ & $(\%)$ & $n b$ & $(\%)$ & $M D$ & $V$ & $Q$ \\
\hline Cl. 1 & & & & & & & \\
$r_{1}$ & 116 & 43.8 & 66 & 24.9 & 12.5 & 9.9 & 14.5 \\
$r_{2}$ & 101 & 38.1 & 34 & 12.8 & 17.3 & 13.3 & 17.0 \\
$r_{3}$ & 48 & 18.1 & 21 & 7.9 & 19.2 & 14.5 & 11.5 \\
$r_{1-3}$ & 265 & 100.0 & 121 & 45.7 & 15.6 & 18.3 & 15.0 \\
\hline Cl. 2 & & & & & & & \\
$r_{1}$ & 62 & 40.5 & 35 & 22.9 & 11.9 & 9.0 & 30.5 \\
$r_{2}$ & 63 & 41.2 & 24 & 15.7 & 20.4 & 13.9 & 30.6 \\
$r_{3}$ & 28 & 18.3 & 10 & 6.5 & 18.3 & 12.1 & 24.5 \\
$r_{1-3}$ & 153 & 100.0 & 69 & 45.1 & 16.6 & 23.5 & 29.4 \\
\hline Cl. 3 & & & & & & & \\
$r_{1}$ & 54 & 27.8 & 41 & 21.1 & 10.3 & 8.3 & 41.3 \\
$r_{2}$ & 47 & 24.2 & 21 & 10.8 & 22.8 & 17.1 & -11.6 \\
$r_{3}$ & 56 & 28.9 & 18 & 9.3 & 31.0 & 22.1 & 8.9 \\
$r_{4}$ & 22 & 11.3 & 10 & 5.2 & 31.7 & 20.1 & 22.9 \\
$r_{5}$ & 15 & 7.7 & 4 & 2.1 & 28.1 & 19.0 & 38.7 \\
$r_{1-5}$ & 194 & 100.0 & 94 & 48.5 & 23.1 & 27.9 & 17.0 \\
\hline Cl. 4 & & & & & & & \\
$r_{1}$ & 28 & 28.0 & 15 & 15.0 & 15.0 & 10.9 & 50.4 \\
$r_{2}$ & 12 & 12.0 & 4 & 4.0 & 17.8 & 12.7 & 53.3 \\
$r_{3}$ & 7 & 7.0 & 4 & 4.0 & 20.6 & 14.4 & 63.9 \\
$r_{4}$ & 15 & 15.0 & 7 & 7.0 & 21.3 & 15.3 & 31.7 \\
$r_{5}$ & 2 & 2.0 & 2 & 2.0 & 6.8 & 3.4 & 81.7 \\
$r_{6}$ & 5 & 5.0 & 2 & 2.0 & 20.7 & 12.0 & 48.4 \\
$r_{7}$ & 4 & 4.0 & 1 & 1.0 & 41.0 & 24.2 & -6.9 \\
$r_{8}$ & 13 & 13.0 & 1 & 1.0 & 29.4 & 13.2 & 39.0 \\
$r_{9}$ & 3 & 3.0 & 1 & 1.0 & 24.3 & 15.1 & 35.3 \\
$r_{10}$ & 6 & 6.0 & 1 & 1.0 & 41.7 & 24.3 & -19.9 \\
$r_{11}$ & 5 & 5.0 & 1 & 1.0 & 37.4 & 20.1 & -6.0 \\
$r_{1-11}$ & 100 & 100.0 & 39 & 39.0 & 22.7 & 37.2 & 39.0 \\
\hline & & & & & & & \\
\hline
\end{tabular}

eleven non redundant sequences from the candidate list. The selected representative set covers nonetheless $39 \%$ of the cases in the cluster while the quality measure reaches its highest level $(Q=39 \%)$. Indeed the discrepancy is high in this group $(V=37.2)$ and representing the sequences with their assigned representative rather than by the center of the set significantly decreases the sum of distances.

\subsection{Comparing sorting criterions}

Table 4 summarizes the outcome obtained with each of the five proposed criteria. The Frequency, Density and Likelihood criteria provide results of quite equivalent quality while the Mean State Frequency (MStFreq) and Centrality criteria are clearly less satisfactory.
Table 4: Comparing criterions with trep $=25 \%$.

\begin{tabular}{|l|r|rr|rr|}
\hline & $n r$ & $n b$ & $(\%)$ & $M D$ & $Q$ \\
\hline Cluster 1 & & & & & \\
Frequency & 3 & 121 & 45.7 & 15.6 & 15.0 \\
Density & 3 & 121 & 45.7 & 15.6 & 15.0 \\
Likelihood & 3 & 121 & 45.7 & 15.6 & 15.0 \\
MStFreq & 2 & 82 & 30.9 & 25.2 & -37.6 \\
Centrality & 7 & 104 & 39.2 & 18.1 & 1.4 \\
\hline Cluster 2 & & & & & \\
Frequency & 3 & 69 & 45.1 & 16.6 & 29.4 \\
Density & 3 & 69 & 45.1 & 16.6 & 29.4 \\
Likelihood & 2 & 59 & 38.6 & 18.7 & 20.5 \\
MStFreq & 4 & 62 & 40.5 & 18.4 & 21.5 \\
Centrality & 3 & 39 & 25.5 & 29.9 & -27.2 \\
\hline Cluster 3 & & & & & \\
Frequency & 5 & 94 & 48.5 & 23.1 & 17.0 \\
Density & 6 & 100 & 51.5 & 19.3 & 30.6 \\
Likelihood & 8 & 105 & 54.1 & 18.2 & 34.8 \\
MStFreq & 3 & 81 & 41.8 & 31.2 & -12.0 \\
Centrality & 4 & 67 & 34.5 & 31.3 & -12.4 \\
\hline Cluster 4 & & & & & \\
Frequency & 11 & 39 & 39.0 & 22.7 & 39.0 \\
Density & 11 & 37 & 37.0 & 23.8 & 35.9 \\
Likelihood & 7 & 42 & 42.0 & 26.5 & 28.8 \\
MStFreq & 12 & 36 & 36.0 & 29.3 & 21.3 \\
Centrality & 15 & 36 & 36.0 & 29.8 & 19.9 \\
\hline & & & & &
\end{tabular}

Selecting the representative sequences in a candidate list sorted according to the distance to the center yields poor results in many cases. Indeed selecting representatives close from the center of the group leads to poor representation of sequences that are far from it. The sometimes bad results yielded with the Mean State Frequency criterion is attributable to the nature of this criterion, which while focusing on the state frequencies completely ignores their timing.

From Table 4, it seems that among the three winners, Density that is always ranked 1st or $2 \mathrm{nd}$ is the best compromise. We may notice, however, that no criterion yields systematically the smallest number of representatives.

\subsection{Size of the candidate list}

Table 5 presents the results obtained after increasing the trep threshold for the size of the candidate list to $75 \%$. As a consequence the proportion of well represented sequences is now at least $75 \%$. This gain comes however at the cost of a considerable increase in the number $n r$ of selected representative sequences. With this high trep, the 
Table 5: Comparing criterions with trep $=75 \%$.

\begin{tabular}{|l|c|cc|rc|}
\hline & $n r$ & $n b$ & $(\%)$ & $M D$ & $Q$ \\
\hline Cluster 1 & & & & & \\
Frequency & 58 & 224 & 84.5 & 4.4 & 76.0 \\
Density & 58 & 230 & 86.8 & 3.7 & 79.6 \\
Likelihood & 44 & 216 & 81.5 & 5.2 & 71.6 \\
MStFreq & 39 & 201 & 75.8 & 9.1 & 50.2 \\
Centrality & 40 & 202 & 76.2 & 9.4 & 48.5 \\
\hline Cluster 2 & & & & & \\
Frequency & 27 & 129 & 84.3 & 5.0 & 78.9 \\
Density & 26 & 132 & 86.3 & 4.6 & 80.3 \\
Likelihood & 18 & 123 & 80.4 & 6.1 & 74.2 \\
MStFreq & 23 & 122 & 79.7 & 7.1 & 69.6 \\
Centrality & 23 & 119 & 77.8 & 8.5 & 64.0 \\
\hline Cluster 3 & & & & & \\
Frequency & 50 & 158 & 81.4 & 8.2 & 70.7 \\
Density & 58 & 171 & 88.1 & 5.5 & 80.3 \\
Likelihood & 42 & 157 & 80.9 & 8.5 & 69.6 \\
MStFreq & 41 & 148 & 76.3 & 12.3 & 55.9 \\
Centrality & 52 & 156 & 80.4 & 10.4 & 62.6 \\
\hline Cluster 4 & & & & & \\
Frequency & 48 & 87 & 87.0 & 5.6 & 84.9 \\
Density & 48 & 87 & 87.0 & 5.2 & 85.9 \\
Likelihood & 35 & 76 & 76.0 & 10.1 & 72.8 \\
MStFreq & 41 & 77 & 77.0 & 10.8 & 71.0 \\
Centrality & 46 & 76 & 76.0 & 10.1 & 72.8 \\
\hline & & & & \\
& & & &
\end{tabular}

results obtained with the Density criterion get the best scores with any of the three considered quality measures for all four clusters. With Likelihood and Frequency we get results of a quality close to that yielded by Density, while the Mean Sate Frequency and Centrality give again poorer results.

\section{CONCLUSION}

We have presented a flexible method for selecting and visualizing representatives of a set of sequences. The method attempts to find the smallest number of representatives that achieve a given coverage. Different indicators have been considered to measure representativeness and the coverage can be evaluated by means of different sequence dissimilarity measures. The heuristic can be fine tuned with various thresholds for controlling the trade-off between size and quality of the resulting representative set. The experiments demonstrated how good our method is for extracting in an readily interpretable way the main features from sets of sequences. The proposed tools are made available as functions of the TraMineR R-package and are awaiting to be tested with other data sets.

\section{ACKNOWLEDGEMENTS}

This work is part of the Swiss National Science Foundation research project FN-1000015-122230 "Mining event histories: Towards new insights on personal Swiss life courses".

\section{REFERENCES}

Abbott, A. and Forrest, J. (1986). Optimal matching methods for historical sequences. Journal of Interdisciplinary History, 16:471-494.

Abbott, A. and Tsay, A. (2000). Sequence analysis and optimal matching methods in sociology, Review and prospect. Sociological Methods and Research, 29(1):3-33. (With discussion, pp 34$76)$.

Gabadinho, A., Müller, N. S., Ritschard, G., and Studer, M. (2009). Mining sequence data in $R$ with TraMineR: A user's guide. Department of Econometrics and Laboratory of Demography, University of Geneva, Geneva.

Hobohm, U., Scharf, M., Schneider, R., and Sander, C. (1992). Selection of representative protein data sets. Protein Sci, 1(3):409-417.

Holm, L. and Sander, C. (1998). Removing nearneighbour redundancy from large protein sequence collections. Bioinformatics, 14(5):423429.

Kaufman, L. and Rousseeuw, P. J. (1990). Finding groups in data: an introduction to cluster analysis. John Wiley and Sons, New York.

McVicar, D. and Anyadike-Danes, M. (2002). Predicting successful and unsuccessful transitions from school to work by using sequence methods. Journal of the Royal Statistical Society. Series A (Statistics in Society), 165(2):317-334.

Müller, N. S., Gabadinho, A., Ritschard, G., and Studer, M. (2008). Extracting knowledge from life courses: Clustering and visualization. In DAWAK 2008, volume LNCS 5182 of Lectures Notes in Computer Science, pages 176-185, Berlin Heidelberg. Springer.

Studer, M., Ritschard, G., Gabadinho, A., and Müller, N. S. (2009). Analyse de dissimilarités par arbre d'induction. Revue des nouvelles technologies de l'information RNTI, E-15:7-18. 